\begin{tabular}{|c|c|}
\hline Title & W avelength division multiplexing and demultiplexing with photonic crystal waveguide couplers \\
\hline Author(s) & Koshiba, Masanori \\
\hline Citation & $\begin{array}{l}\text { JOURNAL OF LIGHTWA VE TECHNOLOGY, 19(12), 1970-1975 } \\
\text { https://doi.org/10.1109/50.971693 }\end{array}$ \\
\hline Issue Date & 2001-12 \\
\hline Doc URL & http:/hdl.handle.net/2115/5582 \\
\hline Rights & $\begin{array}{l}\text { (0)2001 IEEE. Personal use of this material is permitted. However, permission to reprint/republish this material for } \\
\text { advertising or promotional purposes or for creating new collective works for resale or redistribution to servers or lists, } \\
\text { or to reuse any copyrighted component of this work in other works must be obtained from the IEEE." }\end{array}$ \\
\hline Type & article \\
\hline File Information & JLT19-12.pdf \\
\hline
\end{tabular}

Instructions for use 


\title{
Wavelength Division Multiplexing and Demultiplexing With Photonic Crystal Waveguide Couplers
}

\author{
Masanori Koshiba, Senior Member, IEEE
}

\begin{abstract}
A multiplexer-demultiplexer (MUX-DEMUX) based on PC waveguide couplers is proposed, and its wavelength demultiplexing properties are theoretically investigated. First, a two-channel MUX-DEMUX is designed and characterized, and then, by cascading, two stages of photonic crystal (PC) waveguide couplers with different coupling coefficients are constructed. The device sizes are expected to be drastically reduced from a scale of a few tens of micrometers to a scale of a few hundreds of micrometers in a MUX-DEMUX with a channel spacing of about $20 \mathrm{~nm}$ required for wide-passband wavelength division multiplexing (WWDM) systems. The simulation results are obtained using a time-domain beam propagation method (BPM) based on a finite element scheme and a simpler coupled-mode theory.
\end{abstract}

Index Terms-Beam propagation method (BPM), finite element method, multiplexer-demultiplexer (MUX-DEMUX), photonic crystal, time-domain analysis, wavelength division multiplexing (WDM).

\section{INTRODUCTION}

$\mathbf{P}$ HOTONIC crystals (PCs) [1] have inspired great interest because of their potential ability to control lightwave propagation, and the possibilities of implementing PC-based optical filters into wavelength division multiplexing (WDM) systems have been discussed [2]-[6], focusing on dense-WDM (DWDM) systems for use in point-to-point core networks. DWDM systems require highly accurate wavelength control of the WDM light source and the wavelength multiplexer-demultiplexer (MUX-DEMUX). Recently, a wide-passband WDM (WWDM) system with a relaxed wavelength arrangement has been proposed as a cost-effective approach for access networks offering multiplexed services [7].

In this paper, a MUX-DEMUX based on PC waveguide couplers, especially appropriate for WWDM systems, is proposed, and its wavelength demultiplexing properties are numerically investigated by using a time-domain beam propagation method (TD-BPM) based on a finite element scheme (FETD-BPM) [8] that can employ nonuniform and nonorthogonal meshes. The removal of the fast carrier allows one to track a slowly varying envelope of a pulsed wave directly in time domain and, thus, the converged solution could be obtained with moderate time-step size, compared with the conventional finite-difference time-do-

Manuscript received June 11, 2001; revised August 16, 2001. This work was supported in part by a grant-in-aid for scientific research from the Ministry of Education, Science, and Culture, Japan.

The author is with Division of Electronics and Information Engineering, Hokkaido University, Sapporo 060-8628, Japan.

Publisher Item Identifier S 0733-8724(01)10200-8.

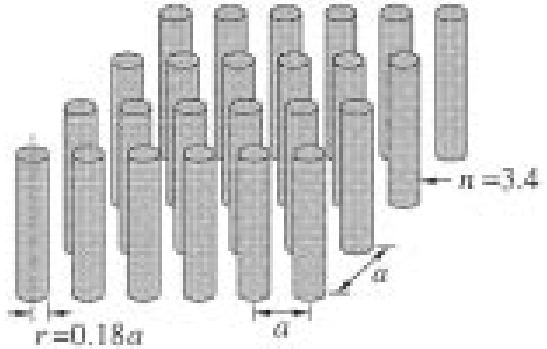

Fig. 1. Photonic crystal.

main method (FDTD). In order to reduce spurious reflections from the computational window edges [9], a high-performance PC-based perfectly matched layer (PML) [10] is introduced.

By the way, a MUX-DEMUX using the conventional directional couplers has been studied for a long time, but the device size is very large. This is because of weak coupling in horizontally arranged couplers. Recently, a MUX-DEMUX using vertical couplers with stronger coupling has been proposed, and a four-channel MUX-DEMUX with 17-nm channel spacing and a two-channel MUX-DEMUX with 11-nm channel spacing have been designed and fabricated [11]. Compared with the conventional couplers, the device sizes become smaller, but still are on the order of millimeters to centimeters.

Another approach for reducing the size of MUX-DEMUX is to use PCs. Here, we present the first demonstration of WDM with PC waveguide couplers. After a two-channel MUX-DEMUX based on PC wave couplers is designed and characterized, a four-channel MUX-DEMUX is constructed by cascading two stages of PC waveguide couplers with different coupling coefficients. The device sizes are drastically reduced from a scale of a few tens of micrometers to a few hundreds of micrometers because of very strong coupling peculiar to PC waveguide couplers.

\section{TWO-CHANNEL MUX-DEMUX}

We consider a PC composed of dielectric pillars in air on square array with lattice constant $a$, as shown in Fig. 1, where the radius and the refractive index of rods are, respectively, taken as $r=0.18 a$ and $n=3.4$. The crystal has a photonic bandgap (PBG) for transverse electric (TE) modes which extends from $\omega a /(2 \pi c)=a / \lambda=0.302$ to 0.443 , but not for transverse magnetic (TM) modes, where $\omega$ is the angular frequency, $c$ the light velocity, and $\lambda$ the wavelength in free space. 


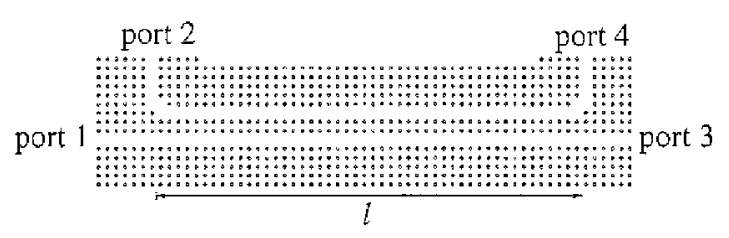

Fig. 2. PC waveguide coupler with two rows of dielectric rods in the interaction region.

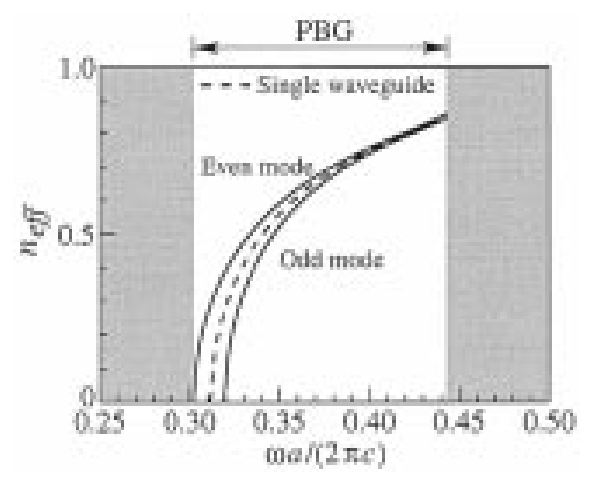

Fig. 3. Dispersion curves of even and odd modes in a PC waveguide coupler with two rows of rods in the interaction region.

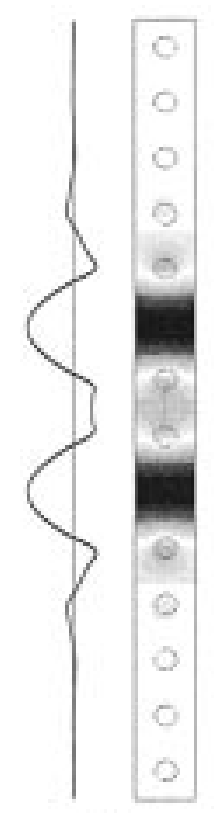

(a)

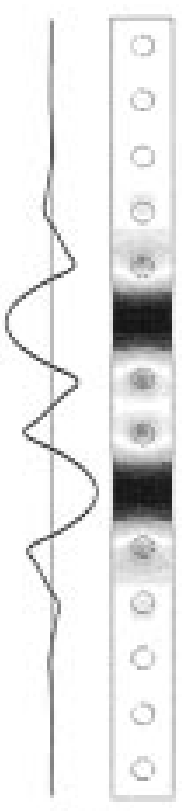

(b)
Fig. 4. Field patterns for (a) even and (b) odd modes in a PC waveguide coupler with two rows of rods in the interaction region.

Now, we propose a two-channel MUX-DEMUX based on a PC waveguide coupler with two rows of dielectric rods in the interaction region, as shown in Fig. 2, where $l$ is the interaction length and in order to separate two input and output waveguides, low-loss $90^{\circ}$ bends [12] are introduced. Gently curved elements such as $S$ bends are not necessary because the PC waveguides with PBG have no radiation loss. Fig. 3 shows the dispersion curves of the guided even and odd modes propagating in the parallel PC waveguides where the dashed line is for the isolated single waveguide. The corresponding field patterns with the transverse profiles on the line defined by the centers of rods are shown in Fig. 4, where $\omega a /(2 \pi c)=0.35$. The difference

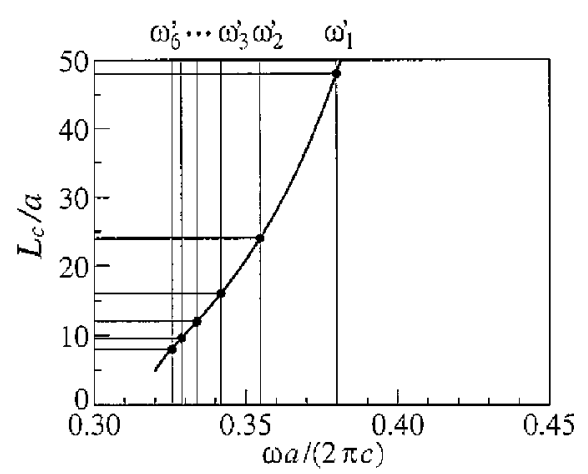

Fig. 5. Coupling length of a PC waveguide coupler with two rows of rods in the interaction region.

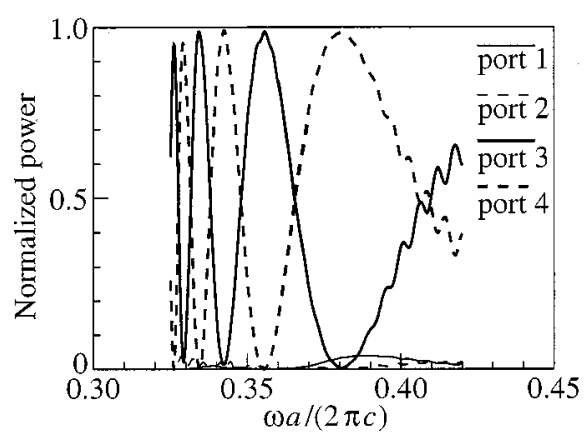

(a)

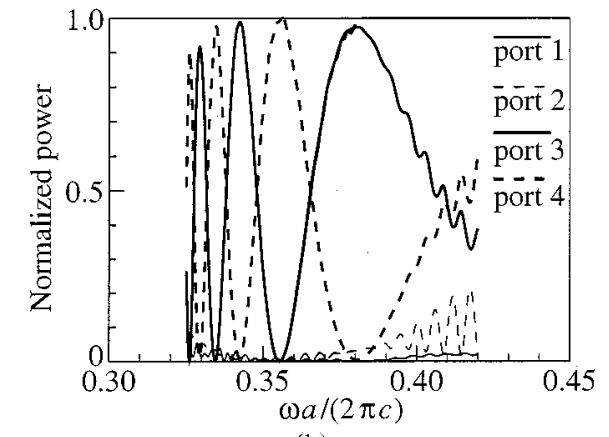

(b)

Fig. 6. Transmission spectrum of a PC waveguide coupler with two rows of rods for input pulses launched into (a) port 1 and (b) port 2.

between the effective indexes of the even and the odd modes is significantly large and, therefore, the coupling length $L_{c}$ is drastically reduced, as shown in Fig. 5, compared with the conventional dielectric waveguide couplers. The transmission spectrum for the structure in Fig. 2 with $l=48 a$ is shown in Fig. 6 where an input pulse with a transverse profile corresponding to the fundamental mode of the isolated single waveguide and a Gaussian profile in the longitudinal direction is launched into port 1 or port 2 . The interaction length of $48 a$ corresponds to the coupling length at frequency of $\omega_{1}^{\prime}$, and is two to six times as large as the coupling lengths at $\omega_{2}^{\prime}$ to $\omega_{6}^{\prime}$, respectively (see Fig. 5).

Fig. 7 shows the field patterns observed in frequency domain. Irrespective of input ports, signals with frequencies of $\omega_{1}^{\prime}$ and $\omega_{3}^{\prime}$ are transferred to the opposite guide (cross state), and signals with frequencies of $\omega_{2}^{\prime}$ and $\omega_{4}^{\prime}$ are outputted from the original guide (bar state). 


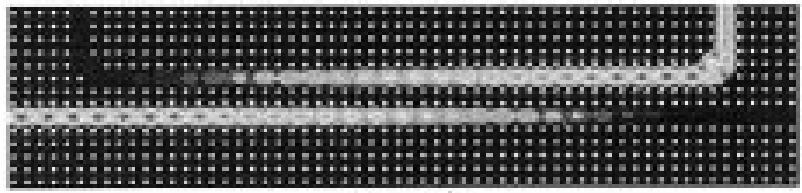

$\omega=\omega_{1}$

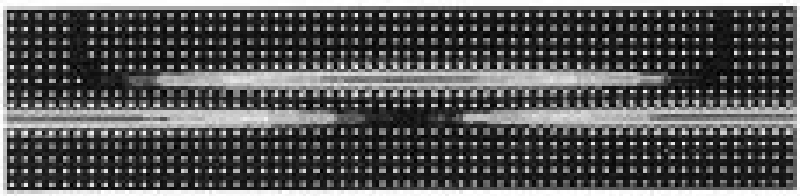

$\omega=\omega^{2}$

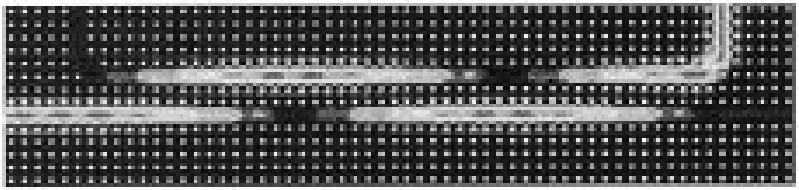

$\omega=\omega_{3}$

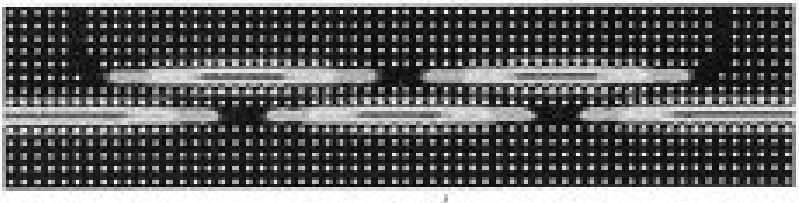

$\omega=\omega t$

(a)

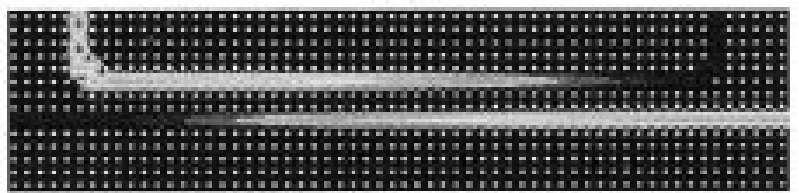

$\omega=\omega_{1}^{\prime}$

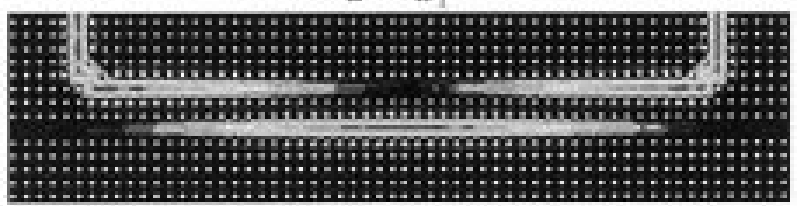

$\omega=\omega_{2}$

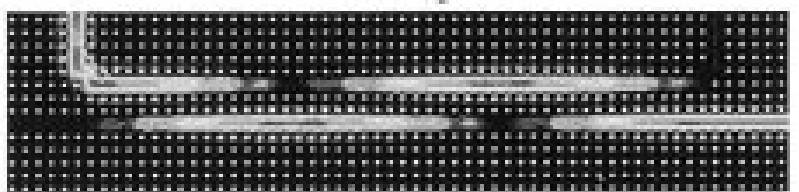

$\omega=\omega_{3}$

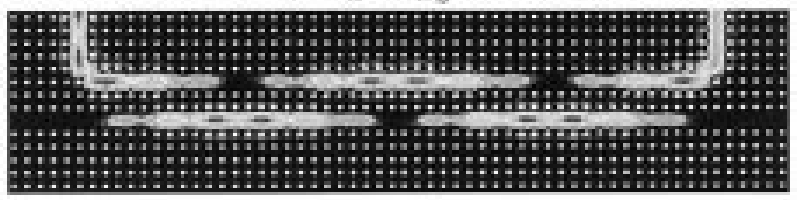

$\omega=\omega_{4}$

(b)

Fig. 7. Field patterns observed in frequency domain for input pulses launched into (a) port 1 and (b) port 2 .

The channel spacing may be further reduced by increasing the device length or the wavelength dependence of the coupling coefficient. Here, as one of the latter examples, we consider a PC waveguide coupler as shown in Fig. 8, where the two straight waveguides are coupled through only one row of rods. Fig. 9 shows the dispersion curves. We can see from the unusual behaviors of the guided even and odd modes that the effective

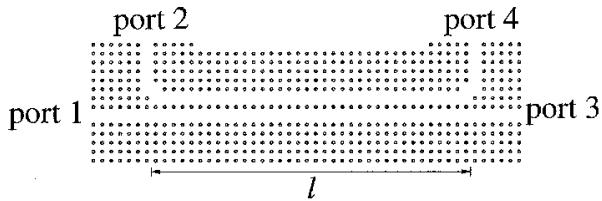

Fig. 8. PC waveguide coupler with one row of dielectric rods in the coupling region in the interaction region.

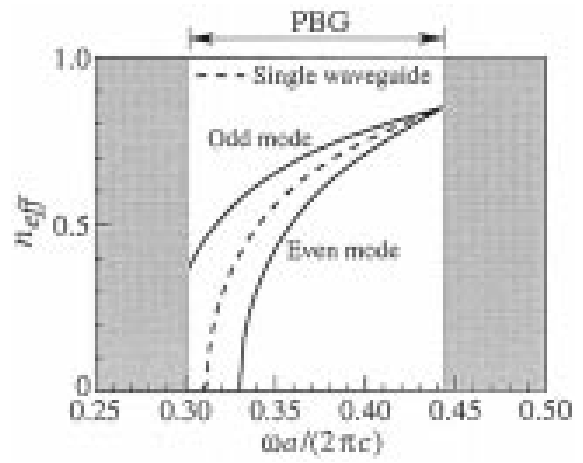

Fig. 9. Dispersion curves of even and odd modes in a PC waveguide coupler with one row of rods in the interaction region.

index of the even mode is smaller than that of the odd mode. This seems to be due to the fact that the modal field of the even mode resembles that of the higher order mode propagating in a conventional five layered dielectric waveguide, as shown in Fig. 10, where $\omega a /(2 \pi c)=0.35$. Fig. 11 shows the coupling length $L_{c}$, which is further reduced, compared with that of the previous coupler with two rows of rods in the interaction region. The transmission spectrum for the structure in Fig. 8 with $l=36 a$ is shown in Fig. 12. As is expected, the channel spacing is reduced, compared with the structure in Fig. 2, but the return loss becomes larger.

\section{FOUR-CHANNEL MUX-DEMUX}

By cascading two stages of PC waveguide couplers with different coupling coefficients, a four-channel MUX-DEMUX is realized, as shown in Fig. 13. The iteration lengths, $l_{1}, l_{2}$, and $l_{3}$, are determined as

$$
\begin{aligned}
l_{1} & =L_{c 2}\left(\omega_{1}\right)=2 L_{c 2}\left(\omega_{2}\right) \\
& =3 L_{c 2}\left(\omega_{3}\right)=4 L_{c 2}\left(\omega_{4}\right) \\
l_{2} & =l_{1} / 2 \\
& =L_{c 2}\left(\omega_{2}\right)=2 L_{c 2}\left(\omega_{4}\right) \\
l_{3} & =m L_{c 1}\left(\omega_{1}\right)=m^{\prime} L_{c 1}\left(\omega_{3}\right)
\end{aligned}
$$

where $L_{c 1}$ and $L_{c 2}$ are the coupling lengths of PC waveguide couplers with one row and two rows of rods in the interaction region, respectively, and the difference between $m$ and $m^{\prime}$ must be odd numbers. Multiplexed signals with frequencies of $\omega_{1}$ to $\omega_{4}$ launched into the left input port are demultiplexed and are outputted from ports $1-4$, respectively.

The interaction lengths are now determined as $l_{1}=44 a, l_{2}=$ $22 a$, and $l_{3}=23.4 a$, based on the data of coupling lengths shown in Fig. 14. As the interaction length normalized by a 


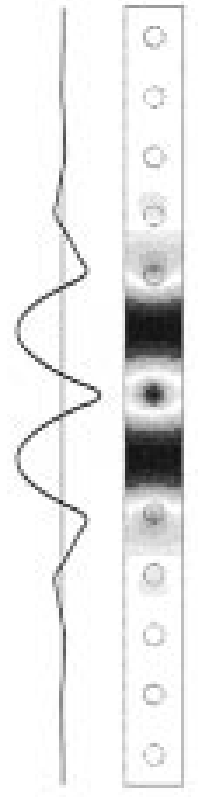

(a)

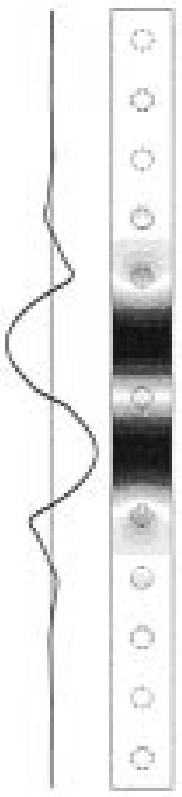

(b)
Fig. 10. Field patterns for (a) even and (b) odd modes in a PC waveguide coupler with one row of rods in the interaction region.

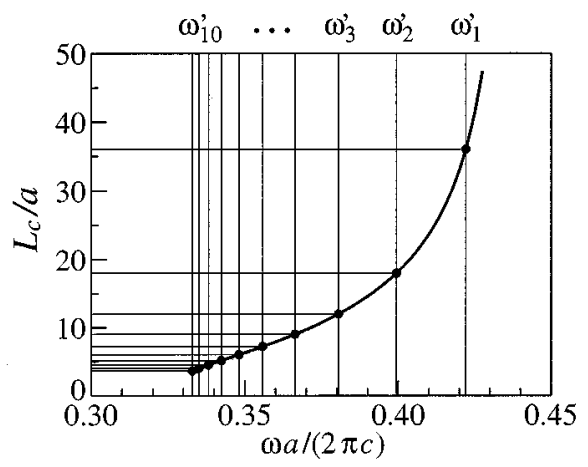

Fig. 11. Coupling length of a PC waveguide coupler with one row of rods in the interaction region.

lattice constant $a$ in a PC waveguide coupler must be an integer, the length $l_{3}$ is approximated as $23 a$. Fig. 15(a) shows the transmission spectrum. The response of port 3 is relatively low, compared with the others. This can be further improved by fine tuning the second stage of PC waveguide couplers. Fig. 15(b) shows the transmission spectrum for $l_{3}=24 a$, resulting in a higher response from port 3.

If the lattice constant is assumed to be $0.54 \mu \mathrm{m}$, the perfect transmission in a $90^{\circ}$ bend [12] is realized at the wavelength of $1.55 \mu \mathrm{m}$, and the device size is about $50 \mu \mathrm{m}$ long and $30 \mu \mathrm{m}$ wide.

\section{Discussion ON CHANNEl SPACING}

Numerical techniques are very powerful and can give accurate results, but large central processing unit times and memory allocations are, in general, required. In order to roughly estimate the interaction length necessary for a desired channel spacing, we use a simpler, well-established coupled-mode theory (CMT).

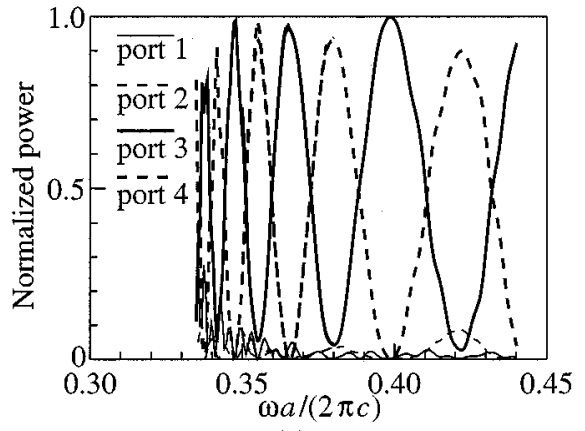

(a)

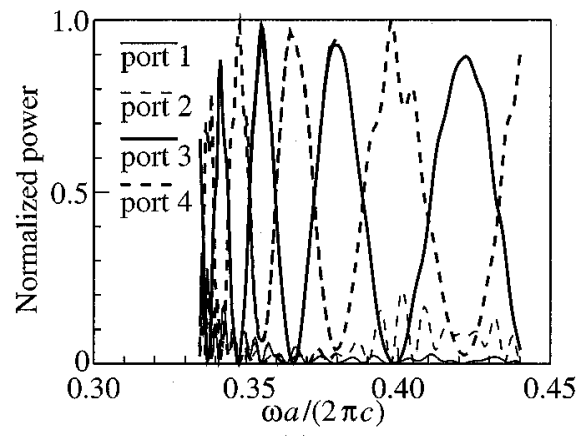

(b)

Fig. 12. Transmission spectrum of a PC waveguide coupler with one row of rods for input pulses launched into (a) port 1 and (b) port 2.

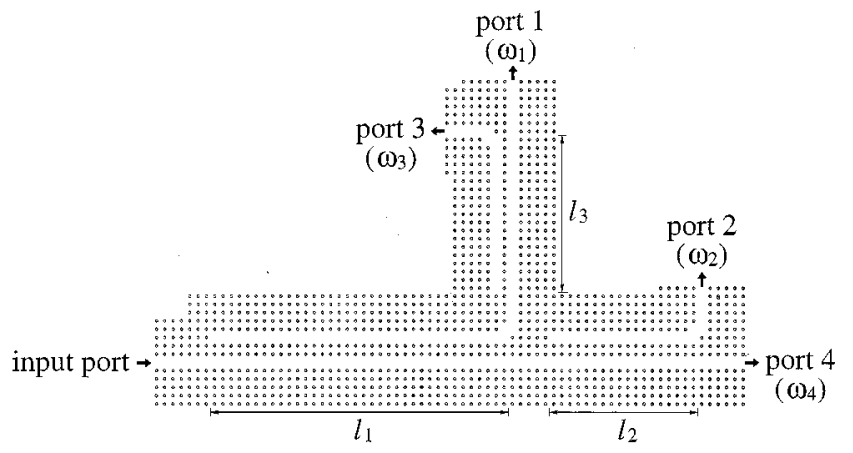

Fig. 13. Four-channel MUX-DEMUX

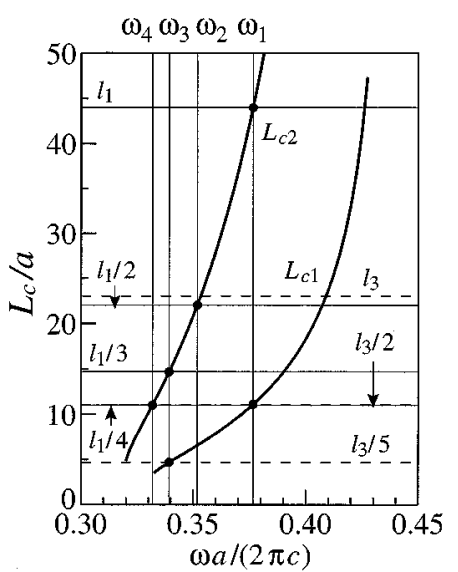

Fig. 14. Coupling lengths of PC waveguide couplers with one row and two rows of rods in the interaction region. 


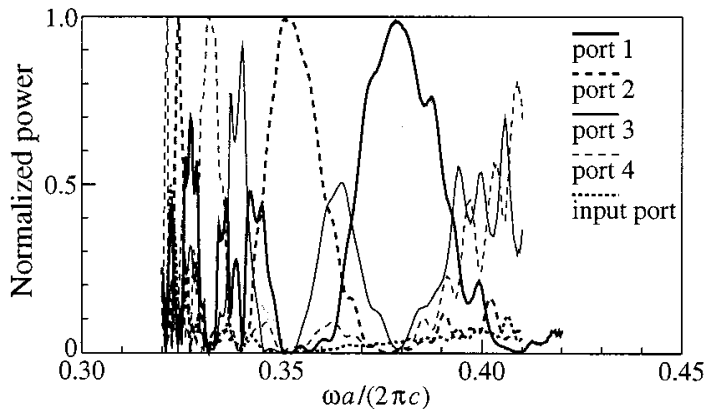

(a)

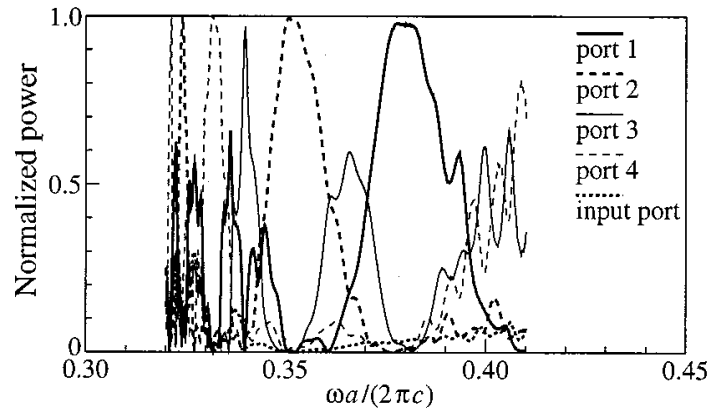

(b)

Fig. 15. Transmission spectrum of a four-channel MUX-DEMUX for (a) $l_{3}=$ $23 a$ and (b) $l_{3}=24 a$ calculated by FETD-BPM.

First, the validity of CMT is checked by reconsidering a fourchannel MUX-DEMUX in Fig. 13. The normalized powers outputted from ports 1-4 are expressed as

$$
\begin{aligned}
& P_{1}=\sin ^{2} \kappa_{2} l_{1} \cos ^{2} \kappa_{1} l_{3} \\
& P_{2}=\cos ^{2} \kappa_{2} l_{1} \sin ^{2} \kappa_{2} l_{2} \\
& P_{3}=\sin ^{2} \kappa_{2} l_{1} \sin ^{2} \kappa_{1} l_{3} \\
& P_{4}=\cos ^{2} \kappa_{2} l_{1} \cos ^{2} \kappa_{2} l_{2}
\end{aligned}
$$

with

$$
\kappa_{i}=\frac{\pi}{2 L_{c i}}=\frac{\left|\beta_{e i}-\beta_{o i}\right|}{2}
$$

where $\kappa_{i}$ is the coupling coefficient, $\beta_{e}$ and $\beta_{o}$ are, respectively, the propagation constants for the even and the odd modes, and the subscripts $i=1$ and 2 stand for the PC waveguide couplers with one row and two rows of rods in the interaction region, respectively.

Fig. 16 shows the transmission spectrum calculated by CMT. With CMT, of course, reflections from $90^{\circ}$ bends and local reflections in periodic PC structures cannot be evaluated. However, channel frequencies and channel spacings are surprisingly in good agreement with the previous results of FETD-BPM in Fig. 15.

Next, a two-channel MUX-DEMUX in Figs. 2 and 8 is considered, and the transmission spectrum is shown in Figs. 17 and 18 , respectively, where the solid and dashed lines are for the bar and cross states, respectively. Assuming the lattice constant $a=0.54 \mu \mathrm{m}$, as described before, the interaction length $l$ for realizing 20-nm channel spacing required in WWDM systems is about $150 a=81 \mu \mathrm{m}$ and $50 a=27 \mu \mathrm{m}$ in the structures in Figs. 2 and 8, respectively.

Even for realizing $0.8-\mathrm{nm}$ channel spacing required in DWDM systems, the interaction length $l$ is only $3000 a=$

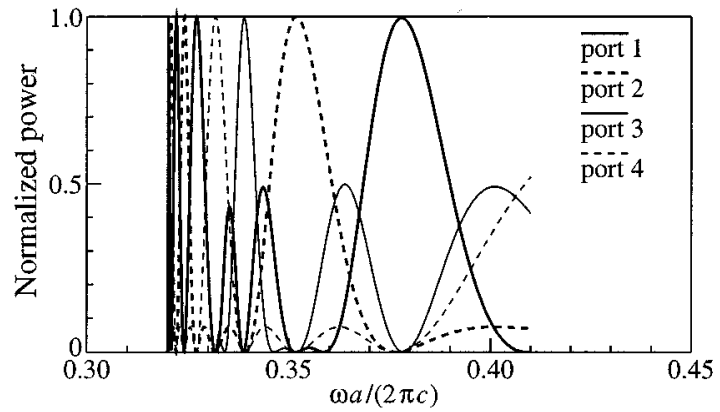

(a)

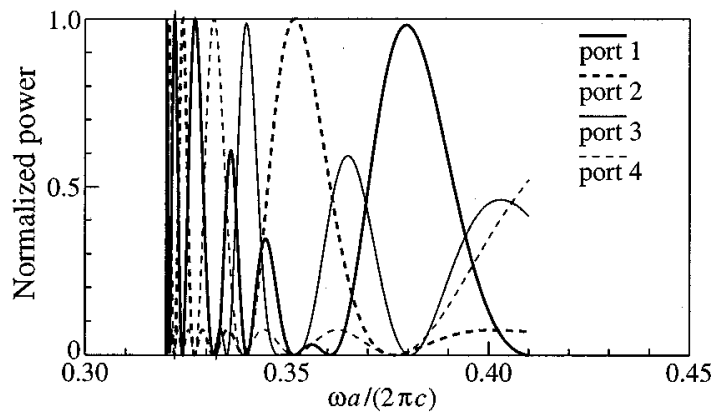

(b)

Fig. 16. Transmission spectrum of a four-channel MUX-DEMUX for (a) $l_{3}=$ $23 a$ and (b) $l_{3}=24 a$ calculated by CMT.

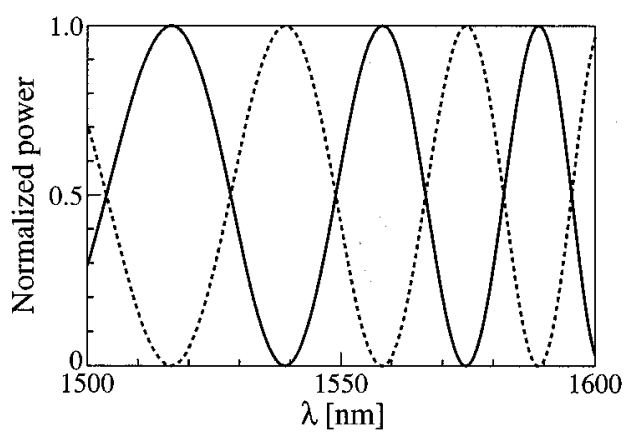

(a)

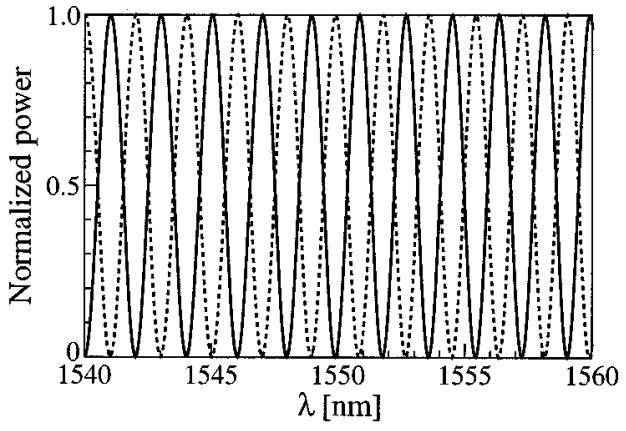

(b)

Fig. 17. Transmission spectrum of a two-channel MUX-DEMUX with iteration length of (a) $l=150 a=81 \mu \mathrm{m}$ and (b) $l=3000 a=1620 \mu \mathrm{m}$ for the structure in Fig. 2.

$1620 \mu \mathrm{m}$ and $1200 a=648 \mu \mathrm{m}$ in the structures in Figs. 2 and 8 , respectively. The channel spacing is approximately proportional to the interaction length.

For both 20-nm and 0.8-nm channel spacings, the interaction length of the structure in Fig. 8 is considerably reduced, compared with the one in Fig. 2. 


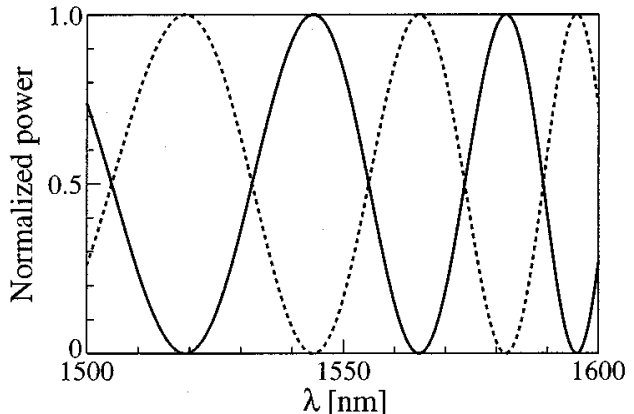

(a)

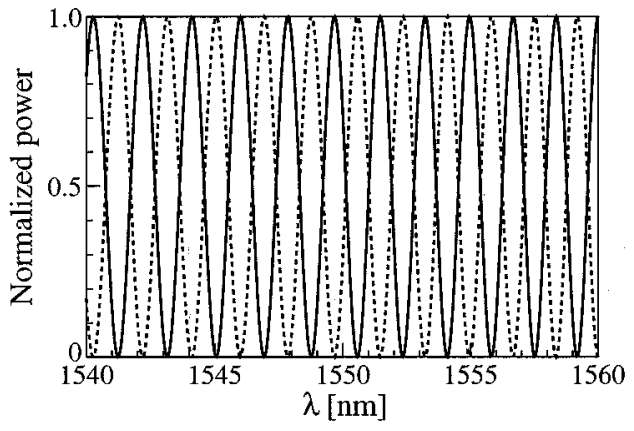

(b)

Fig. 18. Transmission spectrum of a two-channel MUX-DEMUX with iteration length of (a) $l=50 a=27 \mu \mathrm{m}$ and (b) $l=1200 a=648 \mu \mathrm{m}$ for the structure in Fig. 8.

\section{CONCLUSION}

A MUX-DEMUX based on PC waveguide couplers was proposed and its wavelength demultiplexing properties were theoretically investigated using a FETD-BPM method. First, a twochannel MUX-DEMUX was designed and characterized, and then, by cascading two stages of PC waveguide couplers with different coupling coefficients, a four-channel MUX-DEMUX was constructed. In order to roughly estimate the interaction length necessary for a desired channel spacing, a simpler CMT was also introduced. It was confirmed that the device sizes are drastically reduced from a scale of a few tens of micrometers to a few hundreds of micrometers in a MUX-DEMUX with a channel spacing of about $20 \mathrm{~nm}$ required for WWDM systems.

Waveguides created in two-dimensional PCs of disconnected dielectric pillars with infinite length suffer from prohibitive diffraction losses. PCs made of cylindrical pores in a dielectric material seem to be preferable. MUX-DEMUX operations reported here represent a very academic situation in that sense. Further studies on MUX-DEMUX using real, three-dimensional PCs are necessary. Also, a way to couple light efficiently from traditional dielectric waveguides and fibers into and out of PC waveguides should be found [13]-[15]. These subjects are now under consideration.

\section{ACKNOWLEDGMENT}

The author would like to thank Dr. Y. Tsuji of Hokkaido University for interesting discussions and his support during the work, and the reviewer for his very valuable comments.

\section{REFERENCES}

[1] E. Yablonovitch, "Inhibited spontaneous emission in solid-state physics and electronics," Phys. Rev. Lett., vol. 58, pp. 2059-2062, May 1987.
[2] S. Fan, P. R. Villeneuve, J. D. Joannopoulos, and H. A. Haus, "Channel drop tunneling through localized states," Phys. Rev. Lett., vol. 80, pp. 960-963, Feb. 1998.

[3] H. Kosaka, T. Kawashima, A. Tomita, M. Notomi, T. Tamamura, T. Sato, and S. Kawakami, "Photonic crystals for micro lightwave circuits using wavelength-dependent angular beam steering," Appl. Phys. Lett., vol. 74, pp. 1370-1372, Mar. 1999.

[4] E. Centeno, B. Guizal, and D. Felbacq, "Multiplexing and demultiplexing with photonic crystals," J. Opt. A: Pure Appl. Opt., vol. 1, pp. L10-L13, Sept. 1999.

[5] A. Sharkawy, S. Shi, and D. W. Prather, "Multichannel wavelength division multiplexing with photonic crystals," Appl. Opt., vol. 40, pp. 2247-2252, May 2001.

[6] K. Takano and K. Nakagawa, "Frequency analysis of wavelength demultiplexers and optical filters with finite 2-D photonic crystals," IEICE Trans. Electron., vol. E84-C, pp. 669-677, May 2001.

[7] H. Kawata, H. Nakamura, and T. Sugie, "Service multiplexing systems with wide pass band WDM (WWDM) technology for access networks," IEICE Trans. Commun., vol. E83-B, pp. 2348-2354, Oct. 2000.

[8] M. Koshiba, Y. Tsuji, and M. Hikari, "Time-domain beam propagation method and its application to photonic crystal circuits," J. Lightwave Technol., vol. 18, pp. 102-110, Jan. 2000.

[9] A. Mekis, S. Fan, and J. D. Joannopoulos, "Absorbing boundary conditions for FDTD simulations of photonic crystal waveguides," IEEE Microwave Guided Wave Lett., vol. 9, pp. 502-504, Dec. 1999.

[10] M. Koshiba, Y. Tsuji, and S. Sasaki, "High-performance absorbing boundary conditions for photonic crystal waveguide simulations," IEEE Microwave Wireless Compon. Lett., vol. 11, pp. 152-154, Apr. 2001.

[11] B. Liu, A. Shakouri, P. Abraham, and J. E. Bowers, "A wavelength multiplexer using cascaded three-dimensional vertical couplers," Appl. Phys. Lett., vol. 76, pp. 282-284, Jan. 2000.

[12] A. Mekis, J. C. Chen, I. Kurland, S. Fan, P. R. Villeneuve, and J. D. Joannopoulos, "High transmission through sharp bends in photonic crystal waveguides," Phys. Rev. Lett., vol. 77, pp. 3787-3790, Oct. 1996.

[13] Y. Xu, R. K. Lee, and A. Yariv, "Adiabatic coupling between conventional dielectric waveguides and waveguides with discrete translational symmetry," Opt. Lett., vol. 25, pp. 755-757, May. 2000.

[14] A. Adibi, Y. Xu, R. K. Lee, A. Yariv, and A. Scherer, "Properties of the slab modes in photonic crystal optical waveguides," J. Lightwave Technol., vol. 18, pp. 1554-1564, Nov. 2000.

[15] A. Mekis and J. D. Joannopoulos, "Tapered couplers for efficient interfacing between dielectric and photonic crystal waveguides," J. Lightwave Technol., vol. 19, pp. 861-865, June 2001.

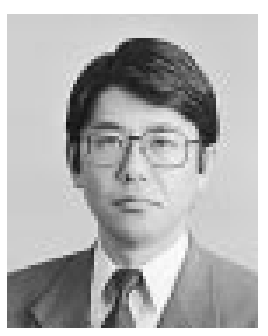

Masanori Koshiba (SM'84) was born in Sapporo, Japan, on November 23, 1948. He received the B.S., M.S., and Ph.D. degrees in electronic engineering from Hokkaido University, Sapporo, Japan, in 1971, 1973, and 1976, respectively.

In 1976, he joined the Department of Electronic Engineering, Kitami Institute of Technology, Kitami, Japan. From 1979 to 1987, he was an Associate Professor of Electronic Engineering at Hokkaido University, and in 1987, he became a Professor there. He has been engaged in research on wave electronics, including microwaves, millimeter waves, lightwaves, surface acoustic waves (SAW), magnetostatic waves (MSW), electron waves, and computer-aided design and modeling of guided-wave devices. He is an author or coauthor of more than 200 research papers in English and of more than 100 research papers in Japanese, both in refereed journals. He authored the books Optical Waveguide Analysis and Optical Waveguide Theory by the Finite Element Method, and coauthored the books Analysis Methods for Electromagnetic Wave Problems, Ultrafast and Ultra-parallel Optoelectronics, and Finite Element Software for Microwave Engineering.

Dr. Koshiba is a member of the Institute of Electronics, Information, and Communication Engineers (IEICE) of Japan, the Institute of Electrical Engineers of Japan, the Institute of Image Information and Television Engineers of Japan, the Japan Society for Simulation Technology, the Japan Society for Computational Methods in Engineering, the Japan Society of Applied Electromagnetics and Mechanics, the Japan Society for Computational Engineering and Science, and the Applied Computational Electromagnetics Society (ACES). He was awarded the 1986, 1996, and 1998 Excellent Paper Awards from the IEICE, and in 1998, he received the Electronics Award from the IEICE Electronics Society. From 1999 to 2000, he served as a President of IEICE Electronics Society, and since 2000, he has been a member of the Board of Directors of ACES. 